\title{
Utilization of Pre-pregnancy Care Services Among Women With High-Risk Pregnancy in the Northern Part of Peninsular Malaysia
}

\author{
Nazirah Jusoh ${ }^{1,2}$, Tengku Alina Tengku Ismail ${ }^{* \mathbb{Q}}$, Noor Aman A. Hamid ${ }^{1}$
}

\begin{abstract}
Objectives: This study aimed to determine the utilization of pre-pregnancy care services and their associated factors among women with high-risk pregnancy in the northern part of Peninsular Malaysia.

Materials and Methods: This cross-sectional study commenced in 2017 among women diagnosed as high-risk pregnancy in governmental health clinics in the state of Perak, Malaysia. Stratified random sampling was applied and interviewer-guided questionnaires were administered in this regard. A proforma and the validated Pre-pregnancy Care Knowledge and Practice Questionnaire were used, consisting 21 items on knowledge and 16 items on practice. The utilization of pre-pregnancy care services was defined if someone had attended the service at least once prior to the current pregnancy. Finally, logistic regression analysis was conducted using SPSS software.

Results: Of 490 women with high-risk pregnancies, $23.9 \%(n=117)$ utilized pre-pregnancy care services. Only one-third of them were aware of the offered services (33.1\%), received advice to seek these services $(30.4 \%)$, and were referred to the services $(27.3 \%)$. There were four significant associated factors for the utilization of pre-pregnancy care services, including maternal age $(P=0.016)$, planned pregnancy $(P<0.001)$, family planning practice $(P<0.001)$, and pre-pregnancy care knowledge $(P=0.030)$.

Conclusions: The utilization of pre-pregnancy care services among women with high-risk pregnancy in this study was low. An increase in maternal age, a planned pregnancy, history of practicing family planning, and women with good pre-pregnancy care knowledge were more likely to utilize pre-pregnancy care services.

Keywords: Pre-pregnancy, Knowledge, High-risk pregnancy, Practice
\end{abstract}

\section{Introduction}

Preconception or pre-pregnancy care is one of the approaches for health promotion and preventive medicine. It covers the aspects of biomedical, behavioral, and social health interventions aiming at improving health and reducing behavioral, individual, and environmental factors that could lead to adverse maternal and child health outcomes (1). Among the advantages of prepregnancy care include the fewer incidence of congenital malformation, stillbirth, and neonatal death in diabetic women, as well as reductions in the incidence of preterm birth, low birth weight, and maternal and neonatal complications among pregnant women in general $(2,3)$. This initiative has been shown to be successful regardless of the income status of the countries (1).

Malaysia has also adapted this initiative since 2002 (4). Women in their reproductive age should receive prepregnancy care before the occurrence of conception. Considering medical problems or risk factors, these women should receive the required care in order to optimize their health conditions at least three months before conception (5). A local study among women screened in pre-pregnancy clinics reported that almost $70 \%$ of them were having at least one risk factor, among whom $35 \%$ were overweight and obese and $14 \%$ of cases had anaemia (6). Thus, pre-pregnancy care is essential for improving pre-pregnancy health status while reducing the risk factors, leading to more favorable maternal and child health outcomes.

Although pre-pregnancy care has been proven as a successful intervention, information on its utilization and knowledge on pre-pregnancy care is inadequate (7). In Malaysia, many women attending pre-pregnancy clinics expressed their initial unawareness of the services and pointed to some neglected areas (8). Thus, it is important to determine its utilization and the level of knowledge and awareness among women in order to improve the service. Accordingly, this study aimed to determine the utilization of pre-pregnancy care services and their associated factors among women with high-risk pregnancy in the northern part of Peninsular Malaysia. 
Key Messages

- Pre-pregnancy care is an important preventive measures for maternal and child health, especially for women in high-risk group.

- Measures should be taken to promote optimal prepregnancy care utilization among women with risk factors, and targeted more on those in young age group.

\section{Materials and Methods}

Study Design and Setting

A cross-sectional study was conducted between March 2016 and March 2017. Perak is one of the states in the northern part of Peninsular Malaysia. There are 12 administrative districts in this state, and the Kinta district with the largest number of population was selected for this study. It has multiracial groups with almost equal proportions of Malay, Chinese and Indian ethnicities. The study commenced in all 14 governmental health clinics with maternal and child health services in this district.

\section{Study Population}

The study population included women diagnosed as having high-risk pregnancies during their first antenatal check-up in governmental health clinics in Kinta District during the study period. The inclusion criteria included pregnant women who came for their first antenatal check-up (booking visit) and were diagnosed as high-risk pregnancy during this visit (4) due to the presence of any of the following issues:

- Current or previous history of hypertension, diabetes, cardiovascular disease, congenital heart disease, thyroid disease, connective tissue disease, systemic lupus erythematosus, chronic kidney disease, preeclampsia, or gestational diabetes;

- Overweight and obese;

- Severe anemia in previous or current pregnancy (A haemoglobin level is less than $7 \mathrm{~g} / \mathrm{dL}$ );

- $\quad$ Age $\leq 19$ years or $>35$ years.

Women who were unable to communicate in either the Malay or English language or those with known mental health problems were excluded from the study.

\section{Sample Size Determination}

The sample size of the study was determined using Power and Sample Size Software for comparing two independent proportions. The proportion of wanted pregnancy was $56.7 \%$ among women who did not utilize pre-pregnancy care (9). The proportion of wanted pregnancy was set at $70.0 \%$ among those who utilized pre-pregnancy care. $\mathrm{Z}_{\alpha}$ and $Z_{\beta}$ were 1.96 and 0.84 for $\alpha=0.05(95 \% \mathrm{CI})$ and $a$ power of $80 \%$, respectively. The ratio $(\mathrm{m})$ between those who utilized and not utilized pre-pregnancy care services was set at 1 . The calculated sample size was 428 , and the required sample size was 492 after 15\% non-response consideration.
Sampling Method

A number of samples proportionate to the size was used by a stratified random sampling technique. There are 14 health clinics with maternal and child health services, and the estimated number of high-risk pregnancy cases in each clinic range from 40 to 100 cases per year. The proportion of selected respondents was based on the percentages of high-risk pregnancies obtained from the registration. Thus, they ranged from a minimum of 30 respondents to a maximum of 45 respondents from every health clinic. These respondents were randomly selected among the pregnant women who fulfilled the study criteria from each health clinic.

\section{Research Tool and Data Collection}

A proforma and the Pre-pregnancy Care Knowledge and Practice Questionnaire were used based on the aim of the study. The proforma consisted of socio-demographic, obstetric data, and pre-pregnancy care information. The utilization of pre-pregnancy care services was defined if someone had attended pre-pregnancy care services at least once prior to the current pregnancy. The information was obtained on how they attended and whether they were referred by the health staff or on their own initiatives. The respondents' awareness of these services was also included in the proforma.

The above-mentioned questionnaire is a validated instrument assessing pre-pregnancy care knowledge and practice (10). The questionnaire was chosen because it was generated in the Malay language and used in another state in Malaysia with the same population ethnic background. Cronbach's alpha of the validated questionnaire was 0.79 . Permission was obtained from the author prior to its use.

The questionnaire has two components of knowledge and practice of pre-pregnancy care. The knowledge domain consisted of 21 items assessing the examples of conditions classified as high-risk pregnancy, birth spacing and impact of poor spacing, anemia, and folic acid, as well as the harmful effects of smoking and healthy diets during pregnancy. The response option used a three-point Likerttype scale (Yes/no/not sure) with a score of "1" for correct answers, and incorrect, and "not sure" responses were scored " 0 ". A total score of more than 12 was categorized as good pre-pregnancy care knowledge and 12 or less was considered as poor knowledge (10). The practice domain consisted of 16 items related to medical check-ups, healthy diets, and healthy lifestyles that women practiced prior to the current pregnancy. Each item had a "Yes/No" option, and a score of " 1 " was given if they answered "Yes" for the recommended practice and "0" for non-recommended practices. A total score of more than 8 was categorized as good pre-pregnancy care practice and 8 or less was considered as poor practice (10).

During data collection, the selected women were informed about the study, and their written consent was obtained accordingly. The interviewer-guided 
questionnaires were administered at a convenient place in the health clinics. On average, answering the questionnaire by the respondents took about 15 minutes.

\section{Statistical Analysis}

Data were entered using IBM SPSS statistics software, version 22. After data entry, it was explored, checked, and cleaned, and a preliminary data description was done to detect any missing value. The data set was checked for errors and corrections were made accordingly. All data were tabulated for descriptive statistics. The categorical variables were summarized as frequency (n) and percentage (\%). In addition, numerical variables were described as the mean and standard deviation (SD) or median and interquartile range (IQR) depending on the normality of distribution. The percentages of women utilizing pre-pregnancy care were calculated as well.

Logistic regression analysis was used to determine factors associated with the utilization of pre-pregnancy care services. The dependent variable was the utilization of the service, coded as " 1 " for "Yes" (utilized) and " 0 " for "No" (not utilized). The variables in the sociodemographic data, obstetric data, as well as the level of pre-pregnancy care knowledge and practice were included as independent variables. The associations between each of these variables with the dependent variable were assessed using simple logistic regression analysis. All variables with $P<0.25$ from this univariable analysis or clinically significant variables were selected based on forward and backward LR methods into multiple logistic regression analysis. The final model was presented by the adjusted odds ratio and 95\% confidence interval (CI), Wald statistics, and $P$ value. The level of significance was set as a $P$ value less than 0.05 .

\section{Results}

A total of 490 women with high-risk pregnancy participated in this study. Table 1 provides the sociodemographic characteristics of the respondents. Their ages ranged from 16 to 43 years old. About 55.7\% had a previous history of complicated pregnancy, of which around $35.0 \%$ experienced miscarriage, gestational diabetes mellitus, and lower segment caesarean section, respectively (Table 2). The majority of them had planned their pregnancy $(60.8 \%)$ although only $24.9 \%$ practiced family planning prior to this pregnancy.

The proportion of women with high-risk pregnancies who utilized pre-pregnancy care services was $23.9 \%$ $(n=117)$. Most of them $(n=106,90.6 \%)$ were referred by the health staff, and only 11 of them attended their own initiatives. Only one-third of them were aware of the offered services (33.1\%), received advice to seek these services (30.4\%), and were referred to the services (27.3\%). Two-hundred and thirty-eight of them (48.6\%) had good pre-pregnancy care knowledge while 376 (76.7\%) of these women had good pre-pregnancy care practice.
Table 1. Socio-demographic Characteristics of Respondents ( $n=490$ )

\begin{tabular}{|c|c|}
\hline Variables & \\
\hline Age (year), Mean (SD) & $29.98(5.51)$ \\
\hline \multicolumn{2}{|l|}{ Ethnicity, No. (\%) } \\
\hline Malay & $347(70.8)$ \\
\hline Chinese & $65(13.3)$ \\
\hline Indian & $74(15.1)$ \\
\hline Others & $4(0.8)$ \\
\hline \multicolumn{2}{|l|}{ Marital status, No. (\%) } \\
\hline Unmarried & $9(1.8)$ \\
\hline Married & $481(98.2)$ \\
\hline \multicolumn{2}{|l|}{ Education level, No. (\%) } \\
\hline Secondary and below & $313(63.9)$ \\
\hline Tertiary & $177(36.1)$ \\
\hline \multicolumn{2}{|l|}{ Employment status, No. (\%) } \\
\hline Unemployed & $217(44.3)$ \\
\hline Employed & $273(55.7)$ \\
\hline Monthly household income (RM) & $3000.00(3000.00)^{\mathrm{a}}$ \\
\hline Distance from health clinic $(\mathrm{km})$ & $3.50(2.50)^{\mathrm{a}}$ \\
\hline \multicolumn{2}{|c|}{ Transportation problems to health clinic, No. (\%) } \\
\hline Yes & $59(12.0)$ \\
\hline No & $431(88.0)$ \\
\hline
\end{tabular}

Note. ${ }^{\mathrm{a}}$ Median (IQR); SD: Standard deviation.

Table 2. Obstetric Characteristics of Respondents $(n=490)$

\begin{tabular}{lc}
\hline Variables & \\
\hline Gravida, median (IQR) & $2.00(2.00)$ \\
Gestational age at booking (wk), median (IQR) & $10.00(5)$ \\
12 weeks and below, No. (\%) & $364(74.3)$ \\
More than 12 weeks, No. (\%) & $126(25.7)$ \\
Previous history of complicated pregnancy, No. (\%) & \\
No & $217(44.3)$ \\
Yes & $273(55.7)$ \\
Types of complicated pregnancy (n=273), No. (\%) & \\
Miscarriage & $98(35.9)$ \\
Induced abortion & $1(0.4)$ \\
Ectopic pregnancy & $7(2.6)$ \\
Gestational diabetes mellitus & $96(35.1)$ \\
Pregnancy-induced hypertension & $46(16.8)$ \\
Congenital heart disease & $2(0.7)$ \\
Cardiovascular disease & $1(0.4)$ \\
Thyroid disease & $8(2.9)$ \\
Renal disease & $3(1.1)$ \\
Postpartum haemorrhage & $1(0.4)$ \\
Perinatal death & $9(3.3)$ \\
Congenital malformation & $368(75.1)$ \\
Instrumental delivery & $122(24.9)$ \\
Caesarian section & $30(11.0)$ \\
Planned pregnancy, No. (\%) & $82(30.0)$ \\
No & \\
Yes & $192(39.2)$ \\
Yes & $298(60.8)$ \\
\hline Tote. SD: Standard deviation; & \\
& \\
The respondents might experience more than one type of complications.
\end{tabular}


Simple logistic regression analysis demonstrated that women's age, employment status, gravida, gestational age at booking, previous complicated pregnancy, planned pregnancy, family planning practice, and pre-pregnancy care knowledge were significantly associated with the utilization of pre-pregnancy care services (Table 3). All variables with $P<0.25$ from this univariable analysis and clinically significant variables were selected for multiple logistic regression analysis using forward and backward LR methods. These variables included women's age, education level, employment status, gravida, gestational age at booking, previous complicated pregnancy, planned pregnancy, family planning practice, and pre-pregnancy care knowledge and practice.
Table 4 presents the multiple logistic regression analysis of factors associated with the utilization of pre-pregnancy care services. Four variables were significantly associated with the utilization of pre-pregnancy care services after controlling for other variables. With one year increase in age, women had 1.07 times odds of utilizing prepregnancy care services when controlled for planned pregnancy, family planning practice, and pre-pregnancy care knowledge (adjusted odds ratio [OR]: 1.07; 95\% CI: $1.01,1.13 ; P=0.016)$. Compared to women who did not plan their pregnancy, those who planned had about three times odds of utilizing pre-pregnancy care services when controlled for age, family planning practice, and pre-pregnancy care knowledge (adjusted OR: 3.14; 95\%

Table 3. Factors Associated With the Utilization of Pre-pregnancy Care Services Using Simple Logistic Regression Analysis $(\mathrm{n}=490)$

\begin{tabular}{|c|c|c|c|c|c|c|}
\hline \multirow[b]{2}{*}{ Variable } & \multicolumn{2}{|c|}{ Utilization of Pre-pregnancy Care } & \multirow[b]{2}{*}{$b$} & \multirow[b]{2}{*}{ Crude OR $(95 \% \mathrm{CI})$} & \multirow[b]{2}{*}{ Wald Statistics (df) } & \multirow[b]{2}{*}{$P$ Value } \\
\hline & $\begin{array}{c}\text { Yes } \\
\text { No }(\%)\end{array}$ & $\begin{array}{c}\text { No } \\
\text { No. }(\%)\end{array}$ & & & & \\
\hline Age (year) & $32.21(4.31)^{\mathrm{a}}$ & $29.21(5.72)^{\mathrm{a}}$ & 0.106 & $1.11(1.07,1.16)$ & $24.70(1)$ & $<0.001$ \\
\hline \multicolumn{7}{|l|}{ Ethnicity } \\
\hline Others & $1(0.9)$ & $3(0.8)$ & & 1 & & \\
\hline Malay & $83(70.9)$ & $264(70.8)$ & -0.06 & $0.94(0.09,9.19)$ & $0.01(1)$ & 0.960 \\
\hline Chinese & $12(10.3)$ & $53(14.2)$ & -0.39 & $0.68(0.07,7.11)$ & $0.10(1)$ & 0.747 \\
\hline Indian & $21(17.9)$ & $53(14.2)$ & 0.17 & $1.19(0.12,12.08)$ & $0.02(1)$ & 0.884 \\
\hline \multicolumn{7}{|l|}{ Marital status } \\
\hline Unmarried & $1(0.9)$ & $8(2.1)$ & & 1 & & \\
\hline Married & $116(99.1)$ & 365 (97.9) & 0.93 & $2.54(0.32,20.54)$ & $0.77(1)$ & 0.381 \\
\hline \multicolumn{7}{|l|}{ Education level } \\
\hline Secondary and below & $69(59.0)$ & $244(65.4)$ & & 1 & & \\
\hline Tertiary & $48(41.0)$ & $129(34.6)$ & 0.27 & $1.32(0.86,2.01)$ & $1.60(1)$ & 0.206 \\
\hline \multicolumn{7}{|l|}{ Employment status } \\
\hline Unemployed & $42(35.9)$ & $175(46.9)$ & & 1 & & \\
\hline Employed & $75(64.1)$ & $198(53.1)$ & 0.46 & $1.58(1.03,2.42)$ & $4.35(1)$ & 0.037 \\
\hline Distance from clinic $(\mathrm{km})$ & $4.78(4.14)^{\mathrm{a}}$ & $4.63(4.47)^{\mathrm{a}}$ & 0.01 & $0.99(0.95,1.04)$ & $0.10(1)$ & 0.749 \\
\hline \multicolumn{7}{|l|}{ Transportation problems } \\
\hline Yes & $14(12.0)$ & $45(12.1)$ & & 1 & & \\
\hline No & $103(88.0)$ & $328(87.9)$ & 0.01 & $1.01(0.53,1.91)$ & $0.01(1)$ & 0.977 \\
\hline Gravida & $3.37(1.46)^{\mathrm{a}}$ & $2.42(1.52)^{\mathrm{a}}$ & 0.37 & $1.45(1.27,1.66)$ & $29.79(1)$ & $<0.001$ \\
\hline \multicolumn{7}{|l|}{ Gestational age at booking } \\
\hline More than 12 weeks & $17(14.5)$ & $109(29.2)$ & & 1 & & \\
\hline 12 weeks and below & $100(85.5)$ & $264(70.8)$ & 0.89 & $2.43(1.39,4.25)$ & $9.63(1)$ & 0.002 \\
\hline \multicolumn{7}{|c|}{ Previous complicated pregnancy } \\
\hline No & $30(25.6)$ & $187(50.1)$ & & 1 & & \\
\hline Yes & $87(74.4)$ & $186(49.9)$ & 1.07 & $2.92(1.84,4.63)$ & $20.61(1)$ & $<0.001$ \\
\hline \multicolumn{7}{|l|}{ Planned pregnancy } \\
\hline No & $19(16.2)$ & $173(46.4)$ & & 1 & & \\
\hline Yes & $98(83.8)$ & $200(53.6)$ & 1.50 & $4.46(2.62,7.59)$ & $30.38(1)$ & $<0.001$ \\
\hline \multicolumn{7}{|l|}{ Family planning practice } \\
\hline No & $38(32.5)$ & $330(88.5)$ & & 1 & & \\
\hline Yes & $79(67.5)$ & $43(11.5)$ & 2.77 & $15.96(9.67,26.32)$ & $117.55(1)$ & $<0.001$ \\
\hline \multicolumn{7}{|c|}{ Pre-pregnancy care knowledge } \\
\hline Poor & $40(34.2)$ & $212(56.8)$ & & 1 & & \\
\hline Good & $77(65.8)$ & $161(43.2)$ & 0.93 & $2.54(1.64,3.91)$ & $17.69(1)$ & $<0.001$ \\
\hline \multicolumn{7}{|l|}{ Pre-pregnancy care practice } \\
\hline Poor & $24(20.5)$ & $90(24.1)$ & & 1 & & \\
\hline Good & 93 (79.5) & $283(75.9)$ & 0.21 & $1.23(0.74,2.05)$ & $0.65(1)$ & 0.420 \\
\hline
\end{tabular}

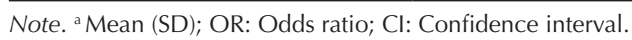


Table 4. Factors Associated With the Utilization of Pre-pregnancy Care Services Using Multiple Logistic Regression Analysis ( $\mathrm{n}=490$ )

\begin{tabular}{|c|c|c|c|c|}
\hline Variable & $b$ & Adjusted OR $(95 \% \mathrm{CI})$ & Wald Statistics $(d f)$ & $P$ Value \\
\hline Age $(y)$ & 0.065 & $1.07(1.01,1.13)$ & $5.75(1)$ & 0.016 \\
\hline \multicolumn{5}{|c|}{ Planned pregnancy } \\
\hline No & & 1 & & \\
\hline Yes & 1.15 & $3.14(1.70,5.81)$ & $13.35(1)$ & $<0.001$ \\
\hline \multicolumn{5}{|c|}{ Family planning practice } \\
\hline No & & 1 & & \\
\hline Yes & 2.45 & $11.55(6.85,19.47)$ & $84.24(1)$ & $<0.001$ \\
\hline \multicolumn{5}{|c|}{ Pre-pregnancy care knowledge } \\
\hline Poor & & 1 & & \\
\hline Good & 0.79 & $2.20(1.30,3.72)$ & $8.61(1)$ & 0.003 \\
\hline
\end{tabular}

Note. OR: Odds ratio; $\mathrm{Cl}$ : confidence interval.

CI: $1.70,5.81 ; P<0.001)$. Compared to those who did not practise family planning prior to this pregnancy, those who practised in this regard had 11 times odds of utilizing prepregnancy care services when controlled for age, planned pregnancy, and pre-pregnancy care knowledge (Adjusted OR: 11.55 ; $95 \%$ CI: $6.85,19.47 ; P<0.001)$. Compared to those with poor pre-pregnancy care knowledge, women with good knowledge had around two times odds of utilizing pre-pregnancy care services when controlled for age, planned pregnancy, and family planning practice (Adjusted OR: 2.20; 95\% CI: 1.30, 3.72; $P=0.03$ ).

\section{Discussion}

Our respondents' mean age was 29.98 (SD: 5.51) years, which is comparable to other similar studies $(11,12)$. However, there were 18 respondents (3.7\%) with the age of 19 years and below. Teenage pregnancy was associated with adverse maternal and perinatal health outcomes, including maternal anemia, preterm birth, low birth weight, and the hypertensive disorders of pregnancy, puerperal endometritis, and systemic infections (1315). Thus, the maternal age of 19 years and below is considered as high-risk pregnancy, together with those in the advanced maternal age.

In this study, the proportion of women was low with a high-risk pregnancy who utilized pre-pregnancy care services. It was even lower than the prevalence among pregnant women in general $(7,9)$. Thus, pre-pregnancy care services should be offered to all women. Nonetheless, those with chronic medical problems, having abnormal body mass index or other risk factors, as well as practicing unhealthy behaviour including smoking and excessive alcohol intake should optimize their health status and modify the behavior before embarking on a pregnancy. Similarly, fertile women in advanced age groups need family planning information and support. These interventions are provided through pre-pregnancy care services. However, a meta-analysis report also showed a low prevalence of pre-pregnancy care service uptake of $34-38 \%$ among women with diabetes (16).
The inadequate utilization of these services could be influenced by many factors. Among the client factors were time constraints, family commitment, poor accessibility, religious issues, and husband's acceptance (8). However, our study also represented that only one-third of women received advice and were referred to the services. A majority of health care providers $(77.0 \%)$ in a study in Malaysia never attended any trainings or courses related to pre-pregnancy care (17). This may affect their practice, particularly in referring the women to these services. However, $89.1 \%$ of them were aware of the existence of the services in their health care settings. Nonetheless, the analysis of in-depth interviews showed their concerns with the lack of training that might affect the efficacy of services (17).

In line with the low percentages of women who received advice or were referred to pre-pregnancy care services, we also found that their knowledge was limited regarding pre-pregnancy care. The low level of knowledge was also reported among women in the reproductive age group, pregnant women, and those with chronic diseases such as diabetes, hypertension, and obesity $(11,18,19)$. A systematic review of pre-pregnancy care studies identified the inadequacies of knowledge among women with regard to the risk of chronic diseases during pregnancy, the behaviour to optimize pre-pregnancy health, and the existence of pre-pregnancy care services (20). Health care providers should be equipped with updated knowledge since they are the main source of information related to pre-pregnancy care and its services. Nonetheless, a local study reported some barriers in pre-pregnancy services among health care providers, including time constraints and workload that limit the opportunity to provide these services, and they were provided by untrained staff due to the lack of human resources (17). This barrier was echoed by the clients of pre-pregnancy clinics as they believed the low knowledge of health care providers on pre-pregnancy care had affected the dissemination of information, and subsequently led to inadequate knowledge among the clients (8). 
Four significant factors of pre-pregnancy care utilization were found in this study, encompassing maternal age, planned pregnancy, family planning practice, and prepregnancy care knowledge. The women were more likely to utilize such services by one year increase in age. Similarly, a study reported that women aged more than 30 years were about three times more likely to utilize the services (21). These women might have thought that their age was no longer suitable for them to get pregnant, especially due to the higher possibility of pregnancy complications, therefore, were more likely to utilize prepregnancy care (12).

The results of our study also indicated that women with planned pregnancies had higher odds to utilize pre-pregnancy care services $(21,22)$. Having an intended pregnancy would motivate women to attain health care before pregnancy (21). They were also more likely to seek medical advice and counselling. Unplanned pregnancy is a major public health concern given that almost half of all pregnancies globally and in Malaysia are unplanned (23). Thus, efforts should be strengthened to reach all women in the reproductive age group and enhance their acceptance of pre-pregnancy care services, which also include family planning and promotion of appropriate inter-pregnancy intervals.

Women who used family planning prior to their current pregnancy were more likely to utilize pre-pregnancy care services. In another study in Malaysia, the bivariate analysis showed that the previous usage of family planning practice had a significant relationship with the attendance to pre-pregnancy care services (7). Additionally, those who never practiced family planning had $85 \%$ low knowledge on pre-pregnancy care as compared to those who had ever used such services (24). Family planning services are provided in the maternal and child health unit, where most pre-pregnancy care services are located. Thus, these women were exposed to more information on pre-pregnancy care services when they came for their family planning follow-ups, subsequently leading to the utilization of these services.

Our study also supported the findings that women with good pre-pregnancy care knowledge had higher odds of utilizing pre-pregnancy care services $(7,12)$. Having good knowledge might have increased their comprehension and realization on the objectives and significance of prepregnancy care, and they were more likely to utilize it (12). A knowledge of pre-pregnancy care is obtained through experience or education (11). Health care providers were the major source of information on pre-pregnancy care (12). Women also believed that there are various measures to promote pre-pregnancy care, including advertisements on television, trains, buses, online information, and leaflets in clinics (25). Accordingly, providing adequate information and ensuring good knowledge of all aspects of pre-pregnancy care are important for increasing the utilization of these services.
In this regard, pre-pregnancy care services should be promoted to ensure that all women in the reproductive age group, especially those with risk factors and medical problems, have adequate awareness and information regarding such services. The role of health care providers is crucial, together with other methods of information dissemination, including mass media and online information. More training is needed for health care providers to equip them with the knowledge and skills to refer women for the services, manage them accordingly, and promote continuous support for these women. In addition, the focus should be given to younger women since they are less likely to utilize these services. Finally, the utilization of pre-pregnancy care services might be improved by focusing on all these factors, and more women might enter the pregnancy state with optimized health.

\section{Strengths and Limitations}

This study had a large distribution of data which involved all health clinics and was conducted in the largest district of Perak. Therefore, it was able to reflect the existing condition related to pre-pregnancy care and can be used by health authorities, for further planning and actions in order to reduce maternal and child health morbidity and mortality by strengthening pre-pregnancy care services. The primary limitation of this study was related to respondents who were recruited from primary health clinics where the offered services are limited. It did not include those who attended preconception care services in government hospitals. This is due to the nature of followups whereby they are managed by hospital specialists throughout their pregnancies. This could have given a different perspective which is more specific toward preconception care in the hospital setting.

\section{Conclusions}

The utilization of pre-pregnancy care services by women with high-risk pregnancies in this study was low. Half of them had good knowledge although their pre-pregnancy practice was satisfactory. An increase in maternal age, a planned pregnancy, history of practicing family planning, and women with good pre-pregnancy care knowledge were among the factors that increased the likelihood of utilizing pre-pregnancy care services.

\section{Authors' Contribution}

$\mathrm{NJ}$ and TATI constructed and designed the study. NJ conducted research and analyzed data. TATI and NAAH reviewed the analysis and the development of the manuscript. All authors have critically reviewed and approved the final draft of the manuscript.

\section{Conflict of Interests}

Authors declare that they have no conflict of interests.

\section{Ethical Issues}

The study was registered with the National Medical Research Register (ID number: NMRR15-1959-28396). The ethical approval was obtained 
from the Human Research Ethics Committee Universiti Sains Malaysia (USM/JEPeM/15090296) and Medical Research and Ethics Committee, the Ministry of Health Malaysia [(7)KKM/NIHSEC/P15-1580], which complies with the Declaration of Helsinki. The study was conducted after obtaining ethical approval from these committees.

\section{Acknowledgments}

We gratefully acknowledge Universiti Sains Malaysia for the research grant (Universiti Sains Malaysia Research University Grant, 1001. PPSP.812212), the Ministry of Health Malaysia for allowing us to conduct the study. Special appreciation also goes to all women who participated, and individuals who were, directly and indirectly, involved in this study.

\section{References}

1. World Health Organization (WHO). Preconception Care: Maximizing the Gains for Maternal and Child Health. Geneva: WHO; 2013.

2. Murphy HR, Roland JM, Skinner TC, et al. Effectiveness of a regional prepregnancy care program in women with type 1 and type 2 diabetes: benefits beyond glycemic control. Diabetes Care. 2010;33(12):2514-2520. doi:10.2337/dc10-1113

3. Jourabchi Z, Sharif S, Lye MS, Saeed A, Khor GL, Tajuddin SHS. Association between preconception care and birth outcomes. Am J Health Promot. 2019;33(3):363-371. doi:10.1177/0890117118779808

4. Ministry of Health Malaysia. Perinatal Care Manual. 3rd ed. Ministry of Health Malaysia; 2013.

5. Ministry of Health Malaysia. Prepregnancy care (PPC). Ministry of Health Malaysia; 2015.

6. Nik Mazlina M, Ruziaton H, Nuraini DB, et al. Risk factors for women attending pre-pregnancy screening in selected clinics in Selangor. Malays Fam Physician. 2014;9(3):20-26.

7. Abu Talib R, Idris IB, Sutan R, Ahmad N, Abu Bakar N. Patterns of Pre-pregnancy Care Usage among Reproductive Age Women in Kedah, Malaysia. Iran J Public Health. 2018;47(11):1694-1702.

8. Rahman M, Rahim NA, Arif MT. Barrier, weakness and utilization of pre-pregnancy clinic services. Arch Public Health. 2017;75:67. doi:10.1186/s13690-017-0236-2

9. Shadab P, Nekuei N, Yadegarfar G. The prevalence of preconception care, its relation with recipients' individuality, fertility, and the causes of lack of checkup in women who gave birth in Isfahan hospitals in 2016. J Educ Health Promot. 2017;6:88. doi:10.4103/ jehp.jehp_99_16

10. Rosnah S, Wan Aisyah W. Pre-pregnancy care among the Indian ethnic group in Semenyih, Selangor. J Community Health. 2010;16(2):29-35

11. Kasim R, Draman N, Abdul Kadir A, Muhamad R. Knowledge, attitudes and practice of preconception care among women attending maternal health clinic in Kelantan. Educ Med J. 2016;8(4):57-68. doi:10.5959/eimj.v8i4.475

12. Demisse TL, Aliyu SA, Kitila SB, Tafesse TT, Gelaw KA, Zerihun MS. Utilization of preconception care and associated factors among reproductive age group women in Debre Birhan town, North Shewa, Ethiopia. Reprod Health. 2019;16(1):96. doi:10.1186/ s12978-019-0758-x

13. Ganchimeg T, Ota E, Morisaki N, et al. Pregnancy and childbirth outcomes among adolescent mothers: a World Health Organization multicountry study. BJOG. 2014;121 Suppl 1:40-48. doi:10.1111/1471-0528.12630

14. Nair A, Devi S. Obstetric outcome of teenage pregnancy in comparison with pregnant women of 20-29 years: a retrospective study. Int J Reprod Contracept Obstet Gynecol. 2015;4(5):13191323. doi:10.18203/2320-1770.ijrcog20150703

15. Yasmin G, Kumar A, Parihar B. Teenage pregnancy-its impact on maternal and fetal outcome. Int J Sci Study. 2014;1(6):9-13.

16. Wahabi HA, Alzeidan RA, Esmaeil SA. Pre-pregnancy care for women with pre-gestational diabetes mellitus: a systematic review and meta-analysis. BMC Public Health. 2012;12:792. doi:10.1186/1471-2458-12-792

17. Abdul Rahim N, Rahman MM. Barriers, strength and weakness of pre-pregnancy clinic services in Sarawak: a qualitative analysis from provider perspectives. Malaysian J Public Health Med. 2018;18(1):149-157.

18. Nepali G, Sapkota SD. Knowledge and practice regarding preconception care among antenatal mothers. International Journal of Perceptions in Public Health. 2017;1(4):224-227.

19. Chuang $\mathrm{CH}$, Velott $\mathrm{DL}$, Weisman CS. Exploring knowledge and attitudes related to pregnancy and preconception health in women with chronic medical conditions. Matern Child Health J. 2010;14(5):713-719. doi:10.1007/s10995-009-0518-6

20. Steel A, Lucke J, Adams J. The prevalence and nature of the use of preconception services by women with chronic health conditions: an integrative review. BMC Womens Health. 2015;15:14. doi:10.1186/s12905-015-0165-6

21. Goshu YA, Liyeh TM, Ayele AS. Preconception care utilization and its associated factors among pregnant women in Adet, NorthWestern Ethiopia (Implication of Reproductive Health). J Womens Health Care. 2018;7(5):445. doi:10.4172/2167-0420.1000445

22. Borges ALV, dos Santos OA, de Castro Nascimento N, do Nascimento Chofakian CB, Gomes-Sponholz FA. Preconception health behaviors associated with pregnancy planning status among Brazilian women. Rev Esc Enferm USP. 2016;50(2):208216. doi:10.1590/s0080-623420160000200005

23. Yusof M, Abdul Samad A, Omar M, Ahmad NA. Unplanned pregnancy and its associated factors. Glob J Health Sci. 2018;10(8):132-142. doi:10.5539/gjhs.v10n8p132

24. Ayalew Y, Mulat A, Dile M, Simegn A. Women's knowledge and associated factors in preconception care in Adet, West Gojjam, Northwest Ethiopia: a community based cross sectional study. Reprod Health. 2017;14(1):15. doi:10.1186/s12978-017-0279-4

25. Barrett G, Shawe J, Howden B, et al. Why do women invest in pre-pregnancy health and care? a qualitative investigation with women attending maternity services. BMC Pregnancy Childbirth. 2015;15:236. doi:10.1186/s12884-015-0672-3

(C) 2021 The Author(s); This is an open-access article distributed under the terms of the Creative Commons Attribution License (http:// creativecommons.org/licenses/by/4.0), which permits unrestricted use, distribution, and reproduction in any medium, provided the original work is properly cited. 